\title{
A novel diagnostic technique to determine uterine health of Holstein cows at 35 days postpartum
}

\author{
V. S. Machado, W. A. Knauer, M. L. S. Bicalho, G. Oikonomou, R. O. Gilbert, and R. C. Bicalho ${ }^{1}$ \\ Department of Population Medicine and Diagnostic Sciences, College of Veterinary Medicine, Cornell University, Ithaca, NY 14853
}

\begin{abstract}
The objectives were (1) to evaluate the association of uterine lavage sample optical density (ULSOD) with uterine health, and (2) to estimate and evaluate a threshold value that will maximize the accuracy of ULSOD as a diagnostic tool for clinical endometritis. The study enrolled 1,742 cows from 3 dairy farms located near Ithaca, New York. The samples were collected at $35 \pm 3 \mathrm{~d}$ in milk (DIM) by using low-volume uterine lavage. Cows with a purulent or mucopurulent secretion in the sample were diagnosed with clinical endometritis, whereas a subgroup of all studied cows was examined for cytological evidence of inflammation by endometrial cytology. Data for ULSOD measured at different wavelengths $(200,352,620,790,860$, and $960 \mathrm{~nm}$ ) were available for 554 cows; all 1,742 cows had data for ULSOD measured at $620 \mathrm{~nm}\left(\mathrm{ULSOD}_{620}\right)$. Incidences of clinical endometritis, metritis, and retained placenta were $10,15.2$, and $5.6 \%$, respectively. The ULSOD 620 was associated with clinical endometritis. Receiver operating characteristic (ROC) analysis of the accuracy of optical density in the detection of clinical endometritis was conducted for ULSOD measured at different wavelengths; ULSOD $_{620}$ was selected for further analysis because it presented the best ROC curve to detect clinical endometritis. The recommended threshold for $\mathrm{ULSOD}_{620}$ ROC was 0.058 , where the sensitivity and specificity were 76.3 and $78.3 \%$, respectively. The ROC analysis of the accuracy of optical density in the detection of endometritis defined as a percentage of neutrophils in the uterine lavage samples higher than $18 \%$ was conducted for $\mathrm{ULSOD}_{620}$. The recommended threshold was 0.059 , where the sensitivity and specificity were 100 and $82.2 \%$, respectively. Cows with $\mathrm{ULSOD}_{620} \leq 0.058$ were 1.21 times more likely to conceive than cows with ULSOD $_{620}>0.058$; moreover, the median calving-to-conception interval for cows that had $\mathrm{ULSOD}_{620} \leq 0.058$ was $122 \mathrm{~d}$ compared with $148 \mathrm{~d}$ for cows that had $\mathrm{ULSOD}_{620}>0.058$. Cows that
\end{abstract}

Received August 24, 2011.

Accepted October 28, 2011.

${ }^{1}$ Corresponding author: rcb28@cornell.edu were positive for Arcanobacterium pyogenes, diagnosed with metritis, or had retained placenta had 4.0, 1.4, and 1.7 times higher odds of having $\mathrm{ULSOD}_{620}>0.058$, respectively. Cows with $\mathrm{ULSOD}_{620}>0.058$ had a higher percentage of neutrophils in the uterine lavage samples than cows with ULSOD $_{620} \leq 0.058$. Uterine lavage sample optical density measured at $620 \mathrm{~nm}$ can be used as an objective indicator of uterine health in dairy cows, principally for clinical endometritis.

Key words: dairy cow, clinical endometritis, uterine disease, metritis

\section{INTRODUCTION}

Reproductive efficiency is undoubtedly a trait of great importance for the dairy industry and significantly affects the overall economic outcome of a dairy enterprise. A healthy reproductive tract is a prerequisite for satisfactory reproductive performance. After parturition, the uterine lumen is usually contaminated by bacteria (Foldi et al., 2006). The complexity of the bacterial community in the postpartum uterus of dairy cows differs between healthy cows and cows with metritis (Santos et al., 2011). Escherichia coli, Arcanobacterium pyogenes, and Fusobacterium necrophorum are considered important etiological agents of uterine diseases (Miller et al., 2007; Bicalho et al., 2010; Santos et al., 2011). Escherichia coli virulence factors, such as fim $H, h l y A, c d t, k p s M I I, i b e A$, and ast $A$, are associated with uterine diseases and impaired reproductive performance (Bicalho et al., 2010). Additionally, F. necrophorum is the most prevalent bacterium in the intrauterine environment of metritic cows, while being completely absent in the uterus of healthy cows (Santos et al., 2011). Furthermore, the presence of A. pyogenes in the uterus is associated with impaired reproduction performance (Williams et al., 2005).

Clinical endometritis is defined as the presence of purulent or mucopurulent uterine exudates in the vagina, after $21 \mathrm{~d}$ postpartum, not accompanied by systemic signs (Sheldon et al., 2006). Although this definition is largely accepted and used by clinicians and researchers, a recent study challenged assumptions of this method of diagnosis, showing that cows with purulent vaginal discharge did not always present endometrial inflam- 
mation (Dubuc et al., 2010a). Clinical endometritis is a disease that affects 15 to $42 \%$ of cows (Plontzke et al., 2010; Potter et al., 2010; Westermann et al., 2010); parity, dystocia, stillbirth, offspring, and metritis are risk factors for the disease (Dubuc et al., 2010b; Potter et al., 2010). The harmful effect of clinical endometritis on reproductive performance (Barlund et al., 2008; Plontzke et al., 2010) and the resulting negative economic impact on the dairy industry (Lee and Kim, 2007) necessitate means for the accurate diagnosis and better investigation and handling of the disease.

Several diagnostic techniques for clinical endometritis are used by researchers, including vaginoscopy, ultrasonographic assessment of uterine fluid volume and endometrial thickness, hysteroscopy, and the use of an intravaginal device (McDougall et al., 2007; Barlund et al., 2008; Madoz et al., 2010). Vaginoscopy and the use of a vaginal mucus collection device (Metricheck, Simcro, Hamilton, New Zealand) are used to evaluate vaginal mucus and diagnose uterine inflammation; however, vaginal discharge can be erroneously associated with uterine inflammation when the discharge is a result of vaginitis or cervicitis. Uterine bacteriology and cytology were used to determine false-positive findings of clinical endometritis by vaginoscopy (Westermann et al., 2010). In addition, ultrasonographic assessment of uterine fluid volume and endometrial thickness was not a good predictor of reproductive performance (Barlund et al., 2008), and hysteroscopy is not a practical technique in the field. Some of these techniques are prone to observer bias and variation between observers, and lack of training could lead to an erroneous diagnosis of clinical endometritis when scoring vaginal discharge by vaginoscopy.

Optical density measurement provides a numerical and objective value of the absorbed light in a sample, and it has been used to measure concentration of cells or proteins in samples (Glasel, 1995; Metris et al., 2006). Uterine lavage sample optical density (ULSOD) might be influenced by the exudates and cellular debris accumulated because of endothelium inflammation (Bondurant, 1999), and by the presence of bacteria inside the uterine lumen of cows affected with endometritis (Miller et al., 2007). Therefore, the objectives were (1) to evaluate the association of ULSOD with uterine health and reproductive performance, and (2) to estimate and evaluate a threshold value that would maximize the accuracy of ULSOD as a diagnostic tool for clinical endometritis.

\section{MATERIALS AND METHODS}

\section{Farms and Management}

The study enrolled 1,742 cows from 3 dairy farms located near Ithaca, New York, from May 4, 2010, until
January 17, 2011. The farms were selected because of their long working relationship with the Ambulatory and Production Medicine Clinic at Cornell University. Farm A milked 3,000 cows, farm B milked 1,600 cows, and farm $\mathrm{C}$ milked 2,800 cows. The cows were housed in freestall barns with concrete stalls covered with mattresses and bedded with waste paper pulp. Cows were milked 3 times daily in milking parlors. All cows were offered a TMR consisting of approximately $55 \%$ forage (corn silage, haylage, and wheat straw) and $45 \%$ concentrate (corn meal, soybean meal, canola, cottonseed, and citrus pulp) on a DM basis of the diet. The diet was formulated to meet or exceed the National Research Council nutrient requirements (NRC, 2001) for lactating Holstein cows weighing $650 \mathrm{~kg}$ and producing $45 \mathrm{~kg}$ of $3.5 \%$ FCM. The reproductive management utilized a combination of Presynch (Moreira et al., 2001), Ovsynch (Pursley et al., 1995), Resynch (Fricke et al., 2003), and detection of estrus, with 25 to $30 \%$ of cows bred via timed AI and the remainder bred after detection of estrus solely by activity monitors (Alpro, DeLaval, Kansas City, MO).

\section{Case Definition, Sample Collection, and OD Measurement}

Clinical endometritis was previously diagnosed primarily by presence of a mucopurulent vaginal discharge (LeBlanc et al., 2002; Sheldon et al., 2006). More recently, it has been established that many cows with purulent or mucopurulent vaginal discharge are free of endometrial inflammation (Dubuc et al., 2010a,b). Clinical endometritis was evaluated at 34 to 36 DIM by visual inspection of a uterine lavage sample obtained as described previously (Gilbert et al., 2005). In this way, visible signs of inflammation (purulent or mucopurulent exudate) emanating from the uterus, rather than from another site, would be assured. Briefly, the cows were restrained, the perineum area was cleansed and disinfected with $70 \%$ ethanol, and a plastic infusion pipette was introduced into the cranial vagina and manipulated through the cervix into the uterus. A total of $20 \mathrm{~mL}$ of sterile saline solution was infused into the uterus and agitated gently, and a sample of the fluid was aspirated. The volume of recovered fluid ranged from 5 to $15 \mathrm{~mL}$. All of the samples were visually scored by one investigator, who assessed the presence of a purulent or mucopurulent secretion in the uterine lavage sample. The score ranged from 0 to 2 , with 0 indicating absence of a purulent or mucopurulent secretion in the lavage sample, 1 indicating a bloody but not purulent sample, and 2 the presence of pus in the lavage sample. Cows with a score of 2 were considered with clinical endometritis. Body condition scores were recorded at 
the time of each uterine lavage using a 5 -point scale (1 $=$ emaciated, $5=$ obese, scored in 0.25 -point intervals) as described by Edmonson et al. (1989). Uterine lavage samples were kept in ice until they were processed in the laboratory and cultured for A. pyogenes on MuellerHinton agar plates (BBL TM 7, Becton, Dickinson and Co., Sparks, MD) supplemented with 5\% defibrinated sheep blood for $48 \mathrm{~h}$ aerobically in $7 \% \mathrm{CO}_{2}$ at $38^{\circ} \mathrm{C}$. Colonies of $A$. pyogenes were distinguished by colony morphology, hemolysis, and Gram stain. After culturing, the samples were frozen at $-80^{\circ} \mathrm{C}$. After all the uterine lavage samples were collected, they were thawed and processed for optical density measurement as follows: hypertonic saline solution $(10 \%)$ was added to the uterine lavage sample in a 1:1 proportion, samples were then incubated at room temperature for $30 \mathrm{~min}$ and subsequently centrifuged for $30 \mathrm{~min}$ at $3,000 \times g$ at $4^{\circ} \mathrm{C}$. The supernatant was collected and an aliquot of $0.2 \mathrm{~mL}$ was added to a 96-well microplate (Greiner Bio-One, Frickenhausen, Germany). The optical density was assessed using a Synergy HT microplate reader (BioTek Instruments, Winooski, VT) and was measured at the following wavelengths for a subset of the study cows (554 cows): 200, 352, 620, 790, 860, and $960 \mathrm{~nm}$. Uterine lavage sample optical density measured at $620 \mathrm{~nm}$ (ULSOD $_{620}$ ) was assessed for all 1,742 cows enrolled in the study. Information regarding parity, calving, presence of retained placenta, and metritis was obtained through the farms' management software.

Additionally, a subgroup $(\mathrm{n}=85)$ of the studied cows was examined for cytological evidence of inflammation with the use of endometrial cytology (Gilbert et al., 2005). Briefly, the uterine lavage samples were mixed by vortex to resuspend cellular material, and an aliquot was transferred to a cytocentrifuge chamber. Samples were centrifuged onto glass slides at $105 \times g$ for 3 min. The slides were then air-dried, and stained with modified Wright-Giemsa stain (Diff-Quik 1, Dade Diagnostics, West Monroe, LA). Coverslips were applied to dried, stained slides using toluene (Permount, Fisher Scientific, Pittsburgh, PA). Each slide was examined by the same examiner under bright-field microscopy at $250 \times$ and $400 \times$. The examiner counted 200 cells from each slide and the percentage of neutrophils among all other cells was estimated. Based on reports from previous studies (Kasimanickam et al., 2004), a threshold of $18 \%$ was used, above which cows were characterized as suffering from endometritis. The $18 \%$ threshold was used because our samples were taken around 35 DIM. Gilbert et al. (2005) suggested a 5\% threshold using samples obtained 40 to 60 DIM.

\section{Statistical Analysis}

To facilitate data analysis and interpretation, the variables BCS group (BCSG), lactation group (LACGP), calf information (CALF), calving ease information (EASE), presence of clinical endometritis at 35 \pm 3 DIM (ENDOM, yes $=1$ and no $=0$ ), presence of metritis during second week of lactation (MET, yes $=1$ and no $=0)$, presence of retained placenta during first week of lactation $(\mathbf{R P}$, yes $=1$ and no $=0)$, and the outcome of the uterine lavage sample culture for A. pyogenes at $35 \pm 3$ DIM (APYO, yes $=1$ and no $=0$ ) were created. Body condition score group was categorized as follows: $\mathrm{BCSG}=1$ if $\mathrm{BCS}<3$, BCSG $=2$ if $\mathrm{BCS}=3$, and $\mathrm{BCSG}=3$ if $\mathrm{BCS}>3$. Lactation group was categorized as follows: LACGP $=1$ if lactation $=1$, LACGP $=2$ if lactation $=2$, and LACGP $=3$ if lactation $>2$. Calf information was categorized as CALF $=1$ if the cow had a live female calf, CALF $=2$ if the cow had a live male calf, $\mathrm{CALF}=3$ if the cow had a stillbirth calf, and CALF $=4$ if the cow had twins. A 5-point scale was used for EASE: EASE 1 was defined as calvings that occurred easily without assistance; EASE 2 was defined as unassisted, but more difficult than EASE 1, calvings; EASE 3 was defined as calvings requiring easy assistance from a person; EASE 4 was defined as vaginally delivered calvings requiring the calf position to be corrected or hard traction to be applied to deliver the calf; and EASE 5 was defined as calvings requiring fetotomy or caesarian section.

Descriptive statistics analysis was undertaken in SAS using the FREQ procedure (SAS Institute Inc., Cary, NC). An ANOVA model was fitted in SAS using the MIXED procedure to assess the effect of several independent variables on $\mathrm{ULSOD}_{620}$. The independent variables BCSG, LACGP, APYO, pregnancy at 100 DIM, pregnancy at 120 DIM, death or culling, CALF, EASE, abortion, RP, and MET were offered to the model. Variables were manually and stepwise removed from the model when the $P$-value $>0.05$. The variable ENDOM was not offered to the model because of its multicollinearity with $\mathrm{ULSOD}_{620}$. All possible 2-way interactions between the independent variables were added to the model; none was significant. To compare the accuracy of OD measured at several different wavelengths $(200,352,620,790,860$, and 960 $\mathrm{nm})$ to predict the dichotomized endometritis score of the uterine lavage sample (ENDOM $=0$ if mucus score $\leq 1, \mathrm{ENDOM}=1$ if mucus score $>1$ ), a receiver operating characteristic (ROC) curve analysis was performed using MedCalc version 11.5.1.0 software 
(MedCalc Software, Mariakerke, Belgium). The area under the curve (AUC) was used to select the most accurate wavelength of OD; $620 \mathrm{~nm}$ had the greatest AUC and was selected for further analysis. To estimate a threshold of ULSOD 620 , a second ROC analysis was performed and a threshold of 0.058 was indicated. In addition to the AUC, the following measures were estimated using MedCalc: sensitivity, specificity, positive predictive value, and negative predictive value. Because it was used for the previous analysis, the gold standard was the dichotomous endometritis diagnosis (endometritis score $>1$ ). A similar ROC analysis was performed with data regarding $\mathrm{ULSOD}_{620}$ and the percentage of neutrophils in uterine lavage samples. In this case, the gold standard was the dichotomous endometritis diagnosis based on information regarding percentage of neutrophils in uterine lavage samples. Cows having a percentage of neutrophils $>18 \%$ were characterized as suffering from endometritis.

The effect of the dichotomized ULSOD ${ }_{620}$ on reproduction was analyzed by Cox's proportional hazard using MedCalc. For this analysis of reproduction, cows were right censored if not diagnosed as being pregnant before culling, death, or the end of the data collection period. Variables offered to the models included dichotomized ULSOD U20 $_{62}$ and LACGP.

A logistic regression was fitted in SAS using the Logistic procedure to examine in detail the association of the independent variables BCSG, LACGP, APYO, pregnancy at 100 DIM, pregnancy at 120 DIM, death or culling, CALF, EASE, abortion, RP and MET with the odds of endometritis as defined by the dichotomized $\mathrm{ULSOD}_{620}$.

Finally, association between clinical endometritis (as defined by visual uterine lavage scores) and dichotomized ULSOD ${ }_{620}$ scores with the cytological evidence of inflammation (measured as the percentage of neutrophils among other cells in uterine lavage samples) were estimated with the use of 1-way ANOVA in JMP 9 (SAS Institute Inc., Cary, NC).

\section{RESULTS}

\section{Descriptive Statistics}

In total, 1,742 cows from 3 farms were enrolled in the study; descriptive statistics concerning the incidence of metritis, retained placenta, and clinical endometritis by lactation, farm and BCSG are in Table 1. Additionally, 29 cows had missing BCS data, and only 554 cows had OD data for all evaluated wavelengths $(200,352,620$, 790, 860, and $960 \mathrm{~nm}$ ). All cows enrolled had data for OD measured at $620 \mathrm{~nm}$.

\section{Effect of Clinical Endometritis, Metritis, A. pyogenes Infection, and Lactation on Measured ULSOD 620}

Uterine lavage sample $\mathrm{OD}_{620}$ was associated with clinical endometritis $(P<0.0001)$. The mean $\mathrm{ULSOD}_{620}$ was 0.18 compared with 0.06 for cows diagnosed with

Table 1. Descriptive statistics of 1,742 cows at study enrollment by lactation, BCS group (BCSG), and farm, including the number of cows per group and the incidence of clinical endometritis, metritis, and retained placenta

\begin{tabular}{|c|c|c|c|c|}
\hline \multirow[b]{2}{*}{ Item } & \multirow[b]{2}{*}{$\begin{array}{c}\text { Cows, } \\
\text { no. }\end{array}$} & \multicolumn{3}{|c|}{ Incidence, \% } \\
\hline & & $\begin{array}{l}\text { Clinical endometritis } \\
\quad(35 \pm 3 \mathrm{DIM})\end{array}$ & $\begin{array}{c}\text { Metritis } \\
\text { (wk } 2 \text { of lactation) }\end{array}$ & $\begin{array}{l}\text { Retained placenta } \\
\text { (wk } 1 \text { of lactation) }\end{array}$ \\
\hline \multicolumn{5}{|c|}{ Lactation } \\
\hline 1 & 565 & 11.4 & 27.1 & 6.4 \\
\hline 2 & 650 & 8.2 & 9.4 & 3.4 \\
\hline$\geq 3$ & 5,527 & 10.6 & 9.7 & 7.6 \\
\hline$P$-value & & 0.14 & $<0.01$ & $<0.01$ \\
\hline Total & 1,742 & 10.0 & 15.2 & 5.6 \\
\hline \multicolumn{5}{|l|}{ BCSG } \\
\hline 1 & 294 & 11.2 & 16.0 & 6.1 \\
\hline 2 & 1,118 & 10.8 & 14.9 & 6.1 \\
\hline 3 & 301 & 6.0 & 15.3 & 3.6 \\
\hline$P$-value & & 0.02 & 0.77 & 0.22 \\
\hline Total & 1,713 & 10.04 & 15.29 & 5.66 \\
\hline \multicolumn{5}{|l|}{ Farm } \\
\hline 1 & 303 & 7.6 & 5.3 & 4.6 \\
\hline 2 & 248 & 6.2 & 8.1 & 8.5 \\
\hline 3 & 1,191 & 11.4 & 19.2 & 5.3 \\
\hline$P$-value & & 0.01 & $<0.01$ & 0.12 \\
\hline Total & 1,742 & 10.0 & 15.2 & 5.6 \\
\hline
\end{tabular}




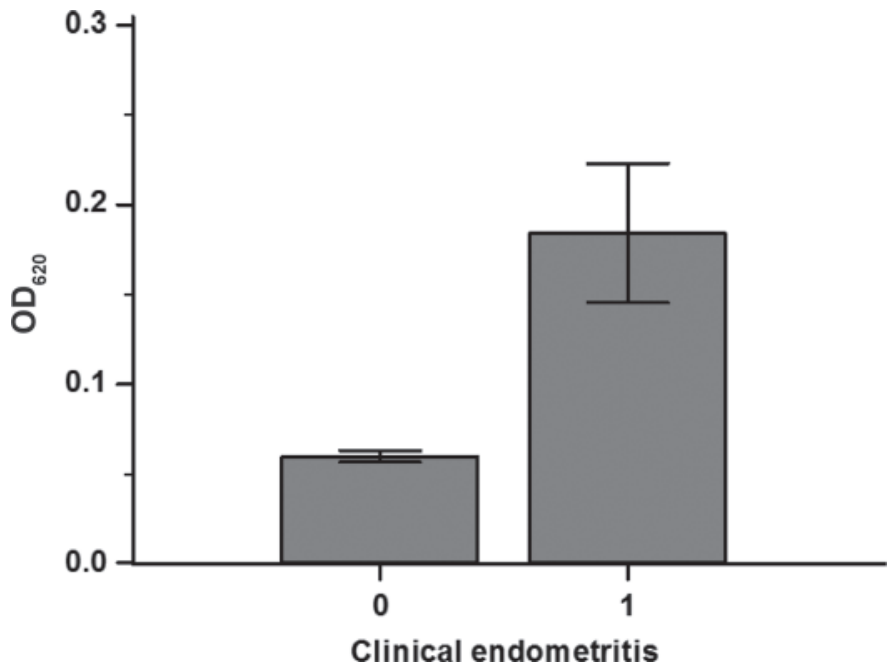

Figure 1. Mean optical density (OD) measured at $620 \mathrm{~nm}$ of uterine lavage samples for cows diagnosed with clinical endometritis (1) and cows diagnosed without clinical endometritis $(0)$. Cows diagnosed with clinical endometritis had mean OD of 0.18 (95\% CI: $0.17-0.20)$ and cows diagnosed without clinical endometritis had mean OD of 0.06 (95\% CI: 0.05-0.07). Error bars represent the 95\% CI for the means $(P<0.0001)$.

and without clinical endometritis, respectively (Figure 1). Clinical endometritis was not subjected to ANOVA.

Uterine lavage sample optical density measured at $620 \mathrm{~nm}$ differed significantly based on lactation, diagnosis of metritis, and presence of intrauterine $A$. pyogenes infection. Cows in lactation 1,2 , and $\geq 3$ had ULSOD $_{620}$ averages of 0.12 (95\% CI: $0.11-0.13$ ), 0.10 (95\% CI: 0.09-0.11), and 0.11 (95\% CI: $0.10-0.12$ ), respectively $(P=0.02)$. In addition, the average UL$\mathrm{SOD}_{620}$ for cows diagnosed with or without metritis was 0.13 (95\% CI: 0.11-0.14) and 0.09 (95\% CI: 0.08-0.10), respectively $(P<0.001)$. Furthermore, cows positive for $A$. pyogenes had a $\mathrm{ULSOD}_{620}$ average of $0.14(95 \%$ CI: $0.12-0.16)$, which was significantly higher than the
ULSOD $_{620}$ of 0.08 (95\% CI: $\left.0.07-0.09\right)$ for cows negative for $A$. pyogenes $(P<0.001)$.

\section{ROC Curve Analysis}

A ROC analysis was performed to evaluate the accuracy of optical density measured at several different wavelengths $(200,352,620,790,860$, and $960 \mathrm{~nm})$. The AUC was $0.84,0.845,0.848,0.830,0.815$, and 0.754 for optical density measured at wavelengths 200, 352, $620,790,860$, and $960 \mathrm{~nm}$, respectively. The ULSOD 620 was selected for further analysis because it presented the greatest AUC between the wavelengths evaluated, and therefore, the best ROC curve to detect clinical endometritis. A second ROC analysis was conducted for $\mathrm{ULSOD}_{620}$ using the data from all cows enrolled, and the AUC for this new ROC curve was 0.85. The recommended threshold for this ROC curve was 0.058 , where the sensitivity was $76.3 \%$ and the specificity $78.3 \%$ (Table 2). A ROC analysis was conducted for $\mathrm{ULSOD}_{620}$ using percentage of neutrophils in uterine lavage samples as the gold standard; cows with $>18 \%$ neutrophils were considered endometritis positive and cows with $\leq 18 \%$ neutrophils were considered endometritis negative. The AUC for this curve was 0.862 . The recommended threshold for this ROC curve was 0.059 , where the sensitivity was $100 \%$ and the specificity $82.2 \%$.

\section{Effect of Dichotomized ULSOD 620 on Reproduction}

A multivariable Cox proportional hazard model was performed, and the only variables retained in this model were LACGP and the dichotomized ULSOD 620 ; cows with $\mathrm{ULSOD}_{620} \leq 0.058$ were 1.21 times more likely to conceive compared with cows with $\mathrm{ULSOD}_{620}$ $>0.058$ (hazard ratio $=1.21, P<0.01$ ). Additionally, the median calving-to-conception interval for cows that

Table 2. Outcome of the receiver operating characteristic (ROC) curve analysis performed to evaluate the optimum uterine lavage sample optical density measured at $620 \mathrm{~nm}$ threshold for the detection of clinical endometritis ${ }^{1}$

\begin{tabular}{|c|c|c|c|c|c|c|}
\hline $\begin{array}{l}\text { Probability } \\
\text { threshold }\end{array}$ & $\begin{array}{l}\text { Sensitivity, \% } \\
(95 \% \text { CI })\end{array}$ & $\begin{array}{l}\text { Specificity, \% } \\
(95 \% \text { CI })\end{array}$ & $\begin{array}{c}\text { Positive predictive } \\
\text { value, } \%\end{array}$ & $\begin{array}{c}\text { Negative predictive } \\
\text { value, } \%\end{array}$ & $\begin{array}{c}\text { Overall } \\
\text { accuracy, \% }\end{array}$ & $\begin{array}{l}\text { Cows above } \\
\text { threshold, \% }\end{array}$ \\
\hline$>0.054$ & $80.9(74.3-86.5)$ & $72.29(70.0-74.5)$ & 24.5 & 97.2 & 73.1 & 33.0 \\
\hline$>0.055$ & $80.3(73.6-86.0)$ & $73.6(71.3-75.7)$ & 25.2 & 97.1 & 74.2 & 31.8 \\
\hline$>0.057$ & $76.3(69.3-82.4)$ & $77.5(75.3-79.5)$ & 27.3 & 96.7 & 77.4 & 27.9 \\
\hline$>0.058^{2}$ & $76.3(69.3-82.4)$ & $78.3(76.1-80.3)$ & 28.0 & 96.7 & 78.1 & 27.2 \\
\hline$>0.059$ & $74.0(66.8-80.4)$ & $79.28(77.2-81.3)$ & 28.4 & 96.5 & 78.7 & 26.0 \\
\hline$>0.062$ & $71.7(64.3-78.3)$ & $82.0(80.0-83.9)$ & 30.6 & 96.3 & 80.9 & 23.4 \\
\hline
\end{tabular}

${ }^{1}$ Sensitivity, specificity, positive predictive value, negative predictive value, overall accuracy, and percentage of cows above the suggested probability threshold were estimated for each hypothetical probability threshold.

${ }^{2}$ Probability threshold suggested by the ROC curve analysis. 


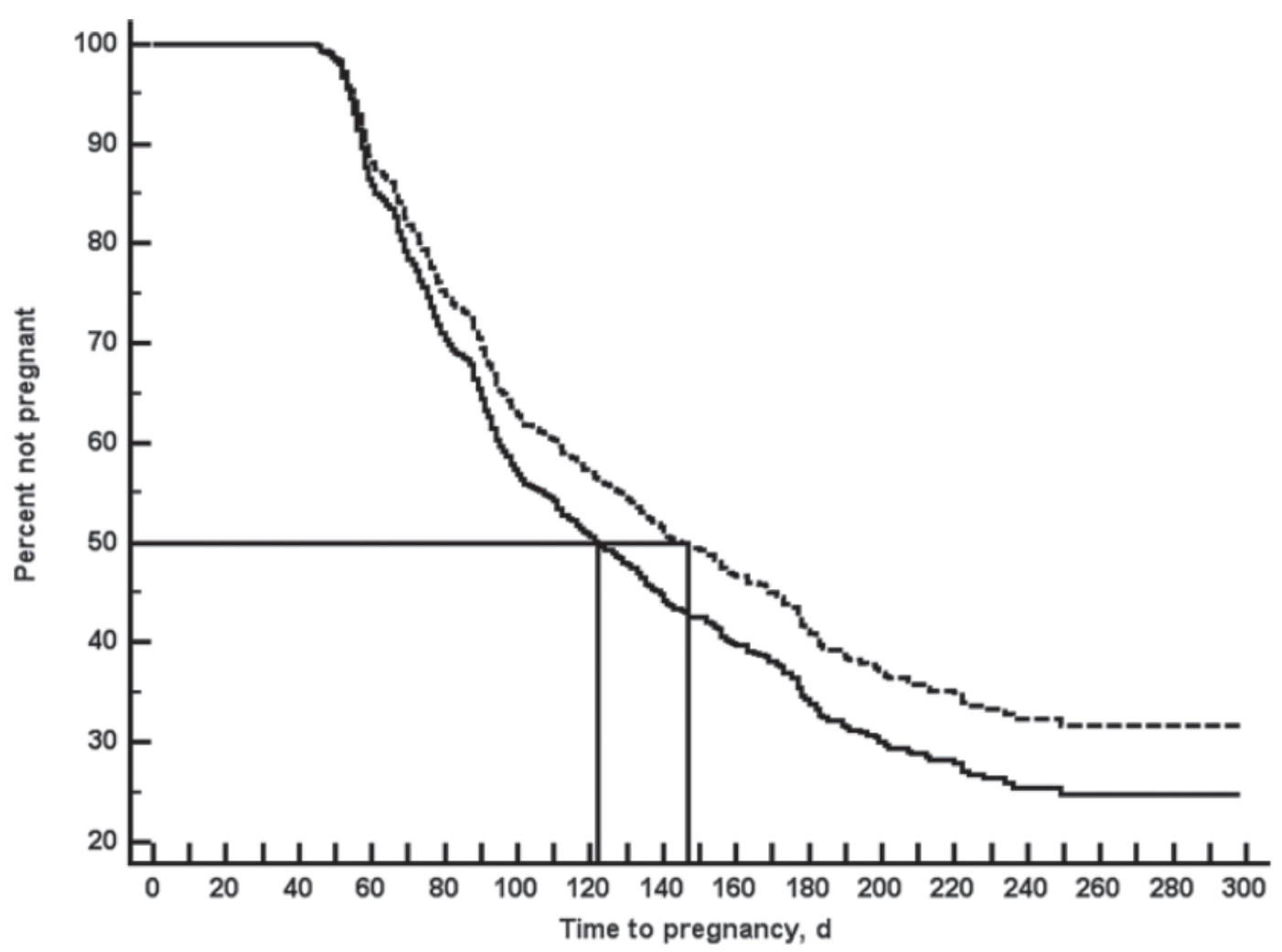

Figure 2. Cox proportional hazards survival analysis of the calving-to-conception interval for cows that presented optical density (OD) measured at $620 \mathrm{~nm}$ of uterine lavage sample $>0.058$. Cows below this threshold (solid line, $\mathrm{n}=1,072$ ) had a median calving-to-conception interval of $122 \mathrm{~d}$ compared with $148 \mathrm{~d}$ for cows that had uterine sample lavage optical density measured at $620 \mathrm{~nm}$ above 0.058 (dashed line, $\mathrm{n}$ $=400)$. Variables offered to the models included dichotomized uterine sample lavage OD measured at $620 \mathrm{~nm}$ and lactation group $(P<0.001)$.

had ULSOD $620 \leq 0.058$ was $122 \mathrm{~d}$ compared with $148 \mathrm{~d}$ for cows with ULSOD $620>0.058$ (Log rank test, $P<$ 0.001 , Figure 2). Moreover, by 250 DIM, the percentage of cows pregnant was significantly lower for cows with ULSOD $_{620}$ above the threshold. By 250 DIM, 68\% of cows with $\mathrm{ULSOD}_{620}>0.058$ were pregnant, whereas $75 \%$ of cows with ULSOD $_{620} \leq 0.058$ had conceived (Figure 2).

\section{Association of Metritis, Retained Placenta, and $A$. pyogenes with the Odds of Endometritis as Defined by the Dichotomized ULSOD 620}

The use of a multivariable logistic regression model revealed that the variables APYO, MET, and RP were significantly associated with the dichotomized UL$\mathrm{SOD}_{620}$. Cows that were positive for APYO had 4.0 times higher odds of having $\mathrm{ULSOD}_{620}>0.058(P<$ $0.001) ; 59.6 \%$ of the APYO positive cows $(\mathrm{n}=151)$ had ULSOD $620>0.058$, and only $24 \%(\mathrm{n}=1,577)$ of the APYO negative cows had ULSOD ${ }_{620}>0.058$. Additionally, cows diagnosed with MET were at a 1.4 increased odds of having $\mathrm{ULSOD}_{620}>0.058(P=0.035) ; 39.6$ and $24.9 \%$ of the cows that were diagnosed with and with- out MET ( $\mathrm{n}=265$ and 1,477$)$ had $\mathrm{ULSOD}_{620}>0.058$, respectively. Moreover, cows with RP had 1.7 times higher odds of having $\mathrm{ULSOD}_{620}$ above the probability threshold suggested by the ROC curve analysis $(26.1 \%$, $\mathrm{n}=1,644 ; P=0.04) ; 44.9 \%$ of the cows with $\mathrm{RP}(\mathrm{n}=$ 98) had ULSOD $620>0.058$.

\section{Association of Clinical Endometritis and Dichotomized ULSOD ${ }_{620}$ Scores with Cytological Evidence of Inflammation}

Results regarding the association of clinical endometritis (as defined by visual uterine lavage scores) and dichotomized ULSOD ${ }_{620}$ scores with the percentage of neutrophils among other cells in uterine lavage samples are in Table 3. Different visual scores or dichotomized ULSOD $_{620}$ scores were accompanied by statistically significant differences in cytological evidence of inflammation. However, the differences were clearer and of greater significance when dichotomized ULSOD $_{620}$ scores were used.

\section{DISCUSSION}

Optical density, also known as absorbance, refers to the mathematical quantity of the physical process of 
Table 3. Association of clinical endometritis and dichotomized uterine lavage sample optical density measured at $620 \mathrm{~nm}\left(\mathrm{ULSOD}_{620}\right)$ with mean percentages of neutrophils in uterine lavage samples

\begin{tabular}{lrc}
\hline Item & Mean $\pm \mathrm{SE}$ & $P$-value \\
\hline Clinical endometritis & & \\
$\quad$ No & $4.4 \pm 1.1$ & 0.013 \\
$\quad$ Yes & $19.5 \pm 5.9$ & \\
ULSOD $_{620}$ & & \\
$\quad \leq 0.058$ & $2.7 \pm 1.2$ & 0.0001 \\
$>0.058$ & $12.6 \pm 2.1$ & \\
\hline
\end{tabular}

absorbing light (Gold and International Union of Pure and Applied Chemistry, 1987). It is largely used to measure the concentration of proteins or cells in suspension in a liquid sample (Glasel, 1995; Metris et al., 2006). In the present study, ULSOD 620 was analyzed by using ROC and visual scoring of clinical endometritis as the gold standard, from which it was determined that a threshold of ULSOD $620>0.058$ should be applied to define diseased cows, achieving an overall accuracy of $78 \%$. It is important to highlight that, currently, no perfect diagnostic test (gold standard) exists for clinical endometritis and, therefore, our ROC analysis was carried out with that limitation.

The incidence of clinical endometritis was $10 \%$, considered low compared with the 15 to $42 \%$ found in other studies (LeBlanc et al., 2002; LeBlanc, 2008; Plontzke et al., 2010). The diagnostic method used in this study may be the main reason for this low incidence of disease. With the low-volume uterine lavage technique, only the presence of a mucopurulent secretion inside the uterus was assessed, eliminating false-positive diagnoses that are more common with other methods of diagnosis such as rectal palpation and visual inspection of vaginal discharge (LeBlanc, 2008; Plontzke et al., 2010; Potter et al., 2010).

Uterine lavage sample optical density measured at $620 \mathrm{~nm}$ was highly associated with the diagnosis of clinical endometritis; the risk factors for high ULSOD $_{620}$ found in this study supported those associated with clinical endometritis found in other studies: presence of $A$. pyogenes in the uterine lumen (Bondurant, 1999), metritis (Dubuc et al., 2010b), and parity (Potter et al., 2010). In contrast, other risk factors described in previous studies were not important for the $\mathrm{ULSOD}_{620}$, such as dystocia, stillbirth, and offspring (Dubuc et al., 2010b; Potter et al., 2010). Retained placenta is a risk factor for clinical endometritis (Potter et al., 2010). In the present study it was not an important risk factor for a high ULSOD 620 value; it was only a risk factor for the dichotomized ULSOD UL20 $_{6}$.

The incidence of retained placenta was $5.6 \%$, which can be considered low when compared with other studies, which reported incidence of 8.6 and $11.8 \%$ (Kelton et al., 1998; Dubuc et al., 2010b). This low incidence could explain why retained placenta was not a risk factor for a high $\mathrm{ULSOD}_{620}$ value. The $\mathrm{ULSOD}_{620}$ mean for cows with retained placenta was greater than that for cows without retained placenta, but because of the low incidence of retained placenta, the statistical power was also low. Nevertheless, retained placenta was significantly important for the dichotomized ULSOD ${ }_{620}$.

Metritis was one of the most important factors associated with $\mathrm{ULSOD}_{620}$. Clinical endometritis and metritis appear to be linked uterine diseases; however, metritis is not necessary for the development of endometritis (Bondurant, 1999; Dubuc et al., 2010b). Arcanobacterium pyogenes can be recovered from the uterus of cows with either normal puerperium or metritis (Silva et al., 2008; Santos et al., 2010), and this infection can persist inside the uterine lumen as endometritis (Bondurant, 1999; Miller et al., 2007). Additionally, clinical endometritis is associated with factors that caused lesions in the uterus, such as dystocia, twin parturition, and retained placenta (Dubuc et al., 2010b; Potter et al., 2010). Therefore, clinical endometritis could be linked to metritis because of the uterine damage caused by metritis (Bondurant, 1999).

The presence of $A$. pyogenes in the uterine lumen has been associated with chronic uterine inflammation (Bondurant, 1999), and thus bacterial culture was used as a means to detect false-positive diagnosis of clinical endometritis when the diagnosis is based on the presence of a mucopurulent discharge in the vagina (Westermann et al., 2010). In the present study, the presence of A. pyogenes in the uterine lavage sample was associated with a high mean $\mathrm{ULSOD}_{620}$. It was reported that A. pyogenes causes severe endometrial lesions (Miller et al., 2007), leading to an antiinflammatory response (Westermann et al., 2010). Therefore, a high ULSOD ${ }_{620}$ could be not only a result of the presence of bacterial cells in the uterine lavage sample, but also the outcome of the endothelial inflammatory process caused by this pathogen. This assumption is supported by the results regarding $\mathrm{ULSOD}_{620}$ and the percentage of neutrophils among other cells in uterine lavage samples. Cows with high $\mathrm{ULSOD}_{620}(>0.058)$ had a significantly higher percentage of neutrophils (Table 3 ).

Endometrial cytology has been used for the diagnosis of endometritis in postpartum Holstein cows. Kasimanickam et al. (2004) defined subclinical endometritis as the presence of $>18 \%$ neutrophils in samples obtained from the uterine lumen of clinically healthy cows at 20 to 33 DIM. The data presented suggested that we could use the dichotomized ULSOD $_{620}$ to accurately (100\% sensitivity, $82.3 \%$ specificity) identify cows with endometritis (defined as the presence of $>18 \%$ neutrophils in samples obtained from the uterine lumen of all 
cows with endometrial cytology information available). Endometrial cytology is considered an objective technique for the diagnosis of endometritis (Gilbert et al., 2005; Westermann et al., 2010). Therefore, ULSOD ${ }_{620}$ measurements could be used as an alternative to endometrial cytology diagnostic techniques, and it should be noted that obtaining $\mathrm{ULSOD}_{620}$ measurements is much less laborious than obtaining endometrial cytology information. The results observed this study must be replicated by other researchers using a distinct population of animals. It should be acknowledged that the $18 \%$ threshold is under discussion because different thresholds have been suggested by other researchers.

Cows diagnosed with endometritis have impaired reproductive performance (Gilbert et al., 2005; Barlund et al., 2008; Plontzke et al., 2010). The extended calving interval and increased culling caused by inefficient reproductive performance cause severe economic loss in the dairy industry (Lee and Kim, 2007). To evaluate the effect of dichotomized $\mathrm{ULSOD}_{620}$ on reproductive performance, a survival analysis was performed (Cox proportional hazard), controlling for parity. Cows with ULSOD $_{620}>0.058$ were less likely to conceive and had a greater median calving-to-conception interval than cows with $\mathrm{ULSOD}_{620} \leq 0.058$. This finding shows that elevated $\mathrm{ULSOD}_{620}$ had a negative effect on reproductive performance and consequently a potential negative economic impact on the dairy herd.

The ULSOD $_{620}$ value could be a useful tool for assessment of uterine health in research. For instance, it could be used as a continuous variable for comparison of different treatment groups. The technique of measuring ULSOD is simple and fast and does not require special training. Moreover, it can prevent diagnostic bias because it provides a numerical value that cannot be influenced by the researcher. It is important that sample collection and processing be standardized to avoid excessive variation between research groups.

In conclusion, we suggest that $\mathrm{ULSOD}_{620}$ could be used for the evaluation of postpartum uterine health. It is a technique that provides objective information of similar quality and accuracy to that obtained by endometrial cytology.

\section{REFERENCES}

Barlund, C. S., T. D. Carruthers, C. L. Waldner, and C. W. Palmer. 2008. A comparison of diagnostic techniques for postpartum endometritis in dairy cattle. Theriogenology 69:714-723.

Bicalho, R. C., V. S. Machado, M. L. Bicalho, R. O. Gilbert, A. G. Teixeira, L. S. Caixeta, and R. V. Pereira. 2010. Molecular and epidemiological characterization of bovine intrauterine Escherichia coli. J. Dairy Sci. 93:5818-5830.

Bondurant, R. H. 1999. Inflammation in the bovine female reproductive tract. J. Anim. Sci. 77(Suppl. 2):101-110.
Dubuc, J., T. F. Duffield, K. E. Leslie, J. S. Walton, and S. J. LeBlanc. 2010a. Definitions and diagnosis of postpartum endometritis in dairy cows. J. Dairy Sci. 93:5225-5233.

Dubuc, J., T. F. Duffield, K. E. Leslie, J. S. Walton, and S. J. LeBlanc. 2010b. Risk factors for postpartum uterine diseases in dairy cows. J. Dairy Sci. 93:5764-5771.

Edmonson, A. J., I. J. Lean, L. D. Weaver, T. Farver, and G. Webster. 1989. A body condition scoring chart for Holstein dairy cows. J. Dairy Sci. 72:68-78.

Foldi, J., M. Kulcsar, A. Pecsi, B. Huyghe, C. de Sa, J. A. Lohuis, P. Cox, and G. Huszenicza. 2006. Bacterial complications of postpartum uterine involution in cattle. Anim. Reprod. Sci. 96:265-281.

Fricke, P. M., D. Z. Caraviello, K. A. Weigel, and M. L. Welle. 2003 Fertility of dairy cows after resynchronization of ovulation at three intervals following first timed insemination. J. Dairy Sci. $86: 3941-3950$.

Gilbert, R. O., S. T. Shin, C. L. Guard, H. N. Erb, and M. Frajblat. 2005. Prevalence of endometritis and its effects on reproductive performance of dairy cows. Theriogenology 64:1879-1888.

Glasel, J. A. 1995. Validity of nucleic acid purities monitored by 260nm/280nm absorbance ratios. Biotechniques 18:62-63.

Gold, V., and International Union of Pure and Applied Chemistry. 1987. Compendium of Chemical Terminology: IUPAC Recommendations. Blackwell Scientific Publications, Oxford, UK.

Kasimanickam, R., T. F. Duffield, R. A. Foster, C. J. Gartley, K. E. Leslie, J. S. Walton, and W. H. Johnson. 2004. Endometrial cytology and ultrasonography for the detection of subclinical endometritis in postpartum dairy cows. Theriogenology 62:9-23.

Kelton, D. F., K. D. Lissemore, and R. E. Martin. 1998. Recommendations for recording and calculating the incidence of selected clinical diseases of dairy cattle. J. Dairy Sci. 81:2502-2509.

LeBlanc, S. J. 2008. Postpartum uterine disease and dairy herd reproductive performance: A review. Vet. J. 176:102-114.

LeBlanc, S. J., T. F. Duffield, K. E. Leslie, K. G. Bateman, G. P. Keefe, J. S. Walton, and W. H. Johnson. 2002. Defining and diagnosing postpartum clinical endometritis and its impact on reproductive performance in dairy cows. J. Dairy Sci. 85:2223-2236.

Lee, J. I., and I. H. Kim. 2007. Pregnancy loss in dairy cows: The contributing factors, the effects on reproductive performance and the economic impact. J. Vet. Sci. 8:283-288.

Madoz, L. V., R. L. De la Sota, K. Suzuki, W. Heuwieser, and M. Drillich. 2010. Use of hysteroscopy for the diagnosis of postpartum clinical endometritis in dairy cows. Vet. Rec. 167:142-143.

McDougall, S., R. Macaulay, and C. Compton. 2007. Association between endometritis diagnosis using a novel intravaginal device and reproductive performance in dairy cattle. Anim. Reprod. Sci. 99:9-23.

Metris, A., S. M. George, and J. Baranyi. 2006. Use of optical density detection times to assess the effect of acetic acid on single-cell kinetics. Appl. Environ. Microbiol. 72:6674-6679.

Miller, A. N., E. J. Williams, K. Sibley, S. Herath, E. A. Lane, J. Fishwick, D. M. Nash, A. N. Rycroft, H. Dobson, C. E. Bryant, and I. M. Sheldon. 2007. The effects of Arcanobacterium pyogenes on endometrial function in vitro, and on uterine and ovarian function in vivo. Theriogenology 68:972-980.

Moreira, F., C. Orlandi, C. A. Risco, R. Mattos, F. Lopes, and W W. Thatcher. 2001. Effects of presynchronization and bovine somatotropin on pregnancy rates to a timed artificial insemination protocol in lactating dairy cows. J. Dairy Sci. 84:1646-1659.

NRC. 2011. Nutrient Requirements of Dairy Cattle. 7 th rev. ed. Natl. Acad. Press, Washington, DC.

Plontzke, J., L. Madoz, R. De la Sota, W. Heuwieser, and M. Drillich. 2010. Prevalence of clinical endometritis and its impact on reproductive performance in grazing dairy cattle in Argentina. Reprod. Domest. Anim. 46:520-526.

Potter, T. J., J. Guitian, J. Fishwick, P. J. Gordon, and I. M. Sheldon. 2010. Risk factors for clinical endometritis in postpartum dairy cattle. Theriogenology 74:127-134.

Pursley, J. R., M. O. Mee, and M. C. Wiltbank. 1995. Synchronization of ovulation in dairy cows using PGF2alpha and GnRH. Theriogenology 44:915-923. 
Santos, T. M., L. S. Caixeta, V. S. Machado, A. K. Rauf, R. O. Gilbert, and R. C. Bicalho. 2010. Antimicrobial resistance and presence of virulence factor genes in Arcanobacterium pyogenes isolated from the uterus of postpartum dairy cows. Vet. Microbiol. 145:84-89.

Santos, T. M., R. O. Gilbert, and R. C. Bicalho. 2011. Metagenomic analysis of the uterine bacterial microbiota in healthy and metritic postpartum dairy cows. J. Dairy Sci. 94:291-302.

Sheldon, I. M., G. S. Lewis, S. LeBlanc, and R. O. Gilbert. 2006. Defining postpartum uterine disease in cattle. Theriogenology 65:1516-1530.

Silva, E., M. Gaivao, S. Leitao, B. H. Jost, C. Carneiro, C. L. Vilela, L. Lopes da Costa, and L. Mateus. 2008. Genomic characterization of Arcanobacterium pyogenes isolates recovered from the uterus of dairy cows with normal puerperium or clinical metritis. Vet. Microbiol. 132:111-118.

Westermann, S., M. Drillich, T. B. Kaufmann, L. V. Madoz, and W. Heuwieser. 2010. A clinical approach to determine false positive findings of clinical endometritis by vaginoscopy by the use of uterine bacteriology and cytology in dairy cows. Theriogenology $74: 1248-1255$

Williams, E. J., D. P. Fischer, D. U. Pfeiffer, G. C. England, D. E. Noakes, H. Dobson, and I. M. Sheldon. 2005. Clinical evaluation of postpartum vaginal mucus reflects uterine bacterial infection and the immune response in cattle. Theriogenology 63:102-117. 